Pacific

Journal of

Mathematics

\title{
MULTIPLE-POINT FORMULAS - A NEW POINT OF VIEW
}

RICHÁRD RIMÁNYI

Volume $202 \quad$ No. 2

February 2002 


\title{
MULTIPLE-POINT FORMULAS - A NEW POINT OF VIEW
}

\author{
RICHÁRD RIMÁNYI
}

\begin{abstract}
On the basis of the Generalized Pontryagin-Thom construction (see Rimanyi \& Szucs, 1998) and its application in computing Thom polynomials (see Rimanyi, 2001) here we introduce a new point of view in multiple-point theory. Using this approach we will first show how to reprove results of Kleiman and his followers (the corank 1 case) then we will prove some new multiple-point formulas which are not subject to the condition of corank $\leq 1$. We will concentrate on the case of complex analytic maps $N^{*} \longrightarrow P^{*+1}$, since this was the setting where the most formulas were known before. The scheme of the computation is similar to the one we used in computing Thom polynomials (see Rimanyi, 2001), with an essential difference that here we need to compute nontrivial incidence classes.
\end{abstract}

\section{Introduction.}

Consider complex analytic maps $f: M^{*} \longrightarrow N^{*+k}(k>0)$. The $*$ is used to put an emphasis on the fact that what follows will not depend on the dimensions of the source and target manifolds, only on their differences. For a nice enough $f$ the closure of the $r$-tuple points of $f$ in the source and in the target determine cohomology classes $m_{r}(f), n_{r}(f)$ - or simply $m_{r}$, $n_{r}$. The so called multiple-point formulas are cohomological identities in the cohomology ring of the source manifold involving these $m_{r}$ 's, $f^{*}\left(n_{r}\right)$ 's and the Chern classes of the map — valid for certain f's. Of course, we like a formula more if it is valid for a bigger set of maps. Such a formula is Ronga's result $m_{2}(f)=f^{*}\left(n_{1}(f)\right)-c_{k}(f)$, which is valid for most maps. Another classical example is the Herbert-Ronga formula $([\mathbf{H}],[\mathbf{R o}])$

$$
m_{r}=f^{*} n_{r-1}-c_{k} m_{r-1}
$$

which is valid only for immersions. In the 80's and 90's it turned out that the Herbert-Ronga formulas can be "corrected" (by adding additional terms involving $c_{i}$ 's for $i>k$ ) so that the new formulas hold for a bigger set of maps - maps of corank $\leq 1$.

The concrete determination of these formulas was a hot area in enumerative algebraic geometry — partly for their own beauty, partly because they 
can be used to obtain other enumerative geometric results, such as e.g., in $[\mathbf{C 1}],[\mathbf{C 2}],[\mathbf{K a} \mathbf{1}],[\mathbf{K a 2}],[\mathbf{J}]$. The modern history of the subject began with works of Laksov, Fulton and Le Barz. The best results are achieved by the two main approaches - i.e., iteration and Hilbert-scheme - of S. Kleiman ([K1], [K2], [K3], [K4], see also [Ka2]) whose papers also contain historical remarks and summaries of how these formulas yield old and new algebraic geometric formulas.

The multiple-point formulas can be considered as the Thom polynomials of multi-singularities (though they are not polynomials). In this paper we show that the approach which turned out to be very powerful in computing (ordinary) Thom polynomials is also capable to find multiple-point formulas. There, and here also, a part of the computation is the determination of certain "incidence (cohomology) classes" (see $[\mathbf{R}]$ ). In fact, the situation here is more difficult, since in the case of Thom polynomials we only had to deal with incidence classes trivially 0 (for geometrical reasons), but in the case of multiple-point formulas we have to compute nontrivial incidence classes. Their computation for the corank 1 singularities leads to the results of $[\mathbf{K} 4]$ and $[\mathbf{K a 2}]$. We can, however, compute incidence classes involving higher corank singularities, and these lead us to some modification of Katz' last formula (now valid not only for corank 1 maps) and some new results.

As we mentioned, the recent research in this area used the techniques of algebraic geometry. Here we use the techniques of "global singularity theory" (= singularity theory + differential topology), the great invention of Szücs, see the introduction and references in $[\mathbf{R S z}]$. Therefore the reader is advised to get some familiarity with the generalized Pontryagin-Thom construction (gPTc) $[\mathbf{R S z}]$ and its application of computing Thom polynomials $[\mathbf{R}]$.

The author is grateful to T. Ohmoto and L. Fehér for helpful discussions, and to OTKA T029759 and FKFP 0226/99 for support.

\section{Main results.}

Following works of Kleiman, Katz [Ka2] proved the following result.

Theorem 2.1. There exist polynomials $p_{i}$ such that for maps $f: M^{*} \longrightarrow$ $N^{*+1}$ of corank $\leq 1$ the following formulas hold modulo torsion

$$
m_{r}=f^{*} n_{r-1}+\sum_{i=1}^{r-1}(-1)^{i} p_{i}(c(f)) m_{r-i} .
$$

So the $p_{i}$ 's are multivariable polynomials evaluated at the Chern classes $c(f)=c\left(f^{*} T N-T M\right)=f^{*} c(N) / c(M)$ of the map.

Since in algebraic geometry some classes are counted with natural multiplicities, so Kleiman's and Katz's $m_{r}$ is $(r-1)$ ! times ours, and their 
$f^{*} f_{*}\left(m_{r}\right)$ is $r$ ! times our $f^{*}\left(n_{r}\right)$. Kleiman also proves so called refined formulas, which assert that the formulas $\left(1_{r}\right)$ really hold, not only modulo torsion.

We will present a new method which reduces the problem of calculating $p_{i}$ 's to solving linear equations. Using this method we will find the following polynomials:

$$
\begin{aligned}
p_{1}= & c_{1} \\
p_{2}= & c_{2} \\
p_{3}= & c_{1} c_{2}+2 c_{3} \\
p_{4}= & c_{1}^{2} c_{2}+c_{2}^{2}+5 c_{1} c_{3}+6 c_{4} \\
p_{5}= & c_{1}^{3} c_{2}+3 c_{1} c_{2}^{2}+9 c_{1}^{2} c_{3}+8 c_{2} c_{3}+26 c_{1} c_{4}+24 c_{5} \\
p_{6}= & c_{1}^{4} c_{2}+6 c_{1}^{2} c_{2}^{2}+2 c_{2}^{3}+14 c_{1}^{3} c_{3}+37 c_{1} c_{2} c_{3}+(56-t) c_{3}^{2}+t c_{2} c_{4} \\
& +71 c_{1}^{2} c_{4}+154 c_{1} c_{5}+120 c_{6} \\
p_{7}= & c_{1}^{5} c_{2}+10 c_{1}^{3} c_{2}^{2}+10 c_{1} c_{2}^{3}+20 c_{1}^{4} c_{3}+105 c_{1}^{2} c_{2} c_{3}+ \\
& 32 c_{2}^{2} c_{3}+\left(362-u_{1}\right) c_{1} c_{3}^{2}+155 c_{1}^{3} c_{4}+ \\
& u_{1} c_{1} c_{2} c_{4}+\left(408-u_{2}\right) c_{3} c_{4}+580 c_{1}^{2} c_{5}+u_{2} c_{2} c_{5}+1044 c_{1} c_{6}+720 c_{7}
\end{aligned}
$$

etc.

Remark 2.2. The polynomials $p_{1}-p_{6}$ appeared in $[\mathbf{K} 4]$ and $[\mathbf{K a} 2]$ with a particular value (42) of the parameter $t$ (in $p_{6}$ ). Here we state that the formulas hold with any value of $t, u_{1}, u_{2}$. Of course, this uncertainty of the polynomials is not a surprise, since the Thom polynomial of the singularity $\mathrm{III}_{2,2}$ (the simplest of Thom-Boardman type $\Sigma^{2}$ ) is $c_{3}^{2}-c_{2} c_{4}$. This explains the presence of the parameter $t$. This, and the Thom polynomial of the singularity $\mathrm{I}_{2,2}$ (the second simplest of type $\Sigma^{2}$ ) - i.e., $c_{1} c_{3}^{2}-c_{1} c_{2} c_{4}+$ $2 c_{3} c_{4}-2 c_{2} c_{5}$ (see $\left.[\mathbf{R}]\right)$ - together explains the presence of the parameters $u_{1}, u_{2}$. Indeed, $u_{1}$ corresponds to $c_{1} \cdot T . P .\left(\mathrm{III}_{2,2}\right)$ and $u_{2}$ corresponds to $\left(T . P .\left(\mathrm{I}_{2,2}\right)-c_{1} \cdot T . P .\left(\mathrm{III}_{2,2}\right)\right) / 2$. These parameters $t, u_{1}, u_{2}$ will take concrete values if we allow the map $f$ to have more difficult singularities (namely $\left.\mathrm{III}_{2,2}, \mathrm{I}_{2,2}\right)$, as follows.

Theorem 2.3. The above formulas hold if $f$ is allowed to have $\mathrm{III}_{2,2}$ and $\mathrm{I}_{2,2}$ singularities, with the following values of the parameters in $p_{6}, p_{7}$

$$
t=43, \quad u_{1}=281, \quad u_{2}=278 .
$$

Of course, allowing a bigger set of maps would be a more spectacular result - and in fact we can allow the maps to have more complicated singularities. Our method, however, works like a test: One can ask, whether the formulas hold if we allow also singularity $\eta$, then we make some algebraic calculation with $\eta$ and answer either yes or no. In fact, for any (stable) singularity the author tried the answer is yes, the formulas hold, therefore here we conjecture that the above formulas hold for any stable maps. The singularities $\mathrm{III}_{2,2}$ and $\mathrm{I}_{2,2}$ are special in the theorem because they are needed to compute the values of the parameters: Allowing $\mathrm{III}_{2,2}$ sets the value of 
$t=43$ and the linear equation $u_{2}=2 u_{1}-284$. Allowing also $\mathrm{I}_{2,2}$ sets the values of $u_{1}$ and $u_{2}$.

\section{A short review on gPTc.}

In this section we give a brief review on the generalized Pontryagin-Thom construction which was invented decades ago by A. Szücs.

Let $k>0$ (in our application $k=1$ ) be fixed and consider the set of all stable germs $\left(\mathbb{C}^{*}, S\right) \longrightarrow\left(\mathbb{C}^{*+k}, 0\right)$ where $S$ is any finite set. Divide this set by the equivalence relation generated by right-left equivalence (permutation of the elements of $S$ are allowed) and trivial unfolding (i.e., adding irrelevant coordinates to the source and to the target). The equivalence classes will be called multi-singularities, or singularities (mono-singularity if $|S|=1$ ). Each singularity has a prototype (defined up to right-left equivalence) such that any other germ of the same singularity is right-left equivalent to an appropriate trivial unfolding of it. The source dimension of the prototype is called the codimension of the singularity. For example, the mono-singularities for $k=1$ up to codim 10 is given in the following table (with their ThomBoardman class):

\begin{tabular}{|c|c|c|c|c|}
\hline codim & $\Sigma^{0}$ & $\Sigma^{1}$ & $\Sigma^{2,0}$ & \\
\hline 0 & $A_{0}$ & & & \\
\hline 1 & & & & \\
\hline 2 & & $A_{1}$ & & \\
\hline 3 & & & & \\
\hline 4 & & $A_{2}$ & & \\
\hline 5 & & & & \\
\hline 6 & & $A_{3}$ & $\mathrm{III}_{2,2}$ & \\
\hline 7 & & & 2,2 & $\mathrm{I}_{2,2}$ \\
\hline 8 & & $A_{4}$ & $\mathrm{III}_{2,3}$ & \\
\hline 9 & & & & $\mathrm{I}_{2,3}$ \\
\hline 10 & & $A_{5}$ & $\mathrm{III}_{2,4} \quad \mathrm{III}_{3,3}$ & \\
\hline
\end{tabular}

Here $A_{i}$ is the unique singularity of Thom-Boardman class $\Sigma^{1_{i}}$ (i.e., the one with local algebra $\left.\mathbb{C}[[x]] /\left(x^{i+1}\right)\right)$. For the singularities with local algebras

$$
\mathbb{C}[[x, y]] /\left(x^{a}, y^{b}, x y\right), \quad \mathbb{C}[[x, y]] /\left(x^{a}+y^{b}, x y\right)
$$

we adapted Mather's notation $\mathrm{III}_{a, b}$ and $\mathrm{I}_{a, b}$, respectively.

We can define the usual hierarchy of singularities: $\eta$ is more complicated than $\zeta(\eta>\zeta)$ if near 0 in the target of a representative of $\eta$ there is necessarily a $\zeta$ point $y$, i.e., the representative at $f^{-1}(y)$ is from $\zeta$. (This is the obvious definition in the case where there is no moduli, so for the sake of simplicity we will stay in that region.) 
Let $\tau$ be an ascending $(\zeta<\eta, \eta \in \tau \Rightarrow \zeta \in \tau$ ) set of singularities. A map $f: N_{0}^{n} \longrightarrow N_{1}^{n+k}$ is called a $\tau$-map if for every $y \in f\left(N_{0}\right)$ the map $f$ near $f^{-1}(y)$ has singularity from $\tau$. For example, $\left\{A_{0}\right\}$-maps are the embeddings, $\left\{r A_{0}\right\}_{r=1,2, \ldots}$-maps are the immersions, or if $\tau$ contains finite linear combinations of $A_{i}$ 's $(i=0,1,2, \ldots)$ then we call a $\tau$-map a Morinmap or a corank 1 map.

In (local) singularity theory one can write up "normal forms" of (prototypes of) singularities. Here we will present a "global normal form" of singularities as follows. For simplicity we give it for a mono-singularity $\eta$ with prototype $\kappa:\left(\mathbb{C}^{n}, 0\right) \longrightarrow\left(\mathbb{C}^{n+k}, 0\right)$. One can consider the maximal compact subgroup of

$$
\text { Aut } \kappa=\left\{(\psi, \phi) \in \operatorname{Diff}\left(\mathbb{C}^{n}, 0\right) \times \operatorname{Diff}\left(\mathbb{C}^{n+k}, 0\right) \mid \phi \circ \kappa \circ \psi^{-1}=\kappa\right\} \text {. }
$$

Denote it by $G_{\eta}$. It comes with two representations $\lambda_{0}, \lambda_{1}$ on the source and the target spaces (which are linear if $\kappa$ is well chosen from its rightleft equivalence class). Now associate vector bundles $\xi_{0}(\eta), \xi_{1}(\eta)$ with the universal principal $G_{\eta}$-bundle using these representations. It can be easily checked that one has a fibrewise map from the total space of $\xi_{0}$ to the total space of $\xi_{1}$ which is (right-left equivalent to) $\kappa$ in each fibre. We call this map $f_{\eta}$ the global normal form of $\eta$.

It is showed in $[\mathbf{R S z}]$ that using the global normal forms of the singularities from $\tau$ one can put together a "universal $\tau$-map" $f \tau: X_{0} \tau \longrightarrow X_{1} \tau-$ from which any $\tau$-map can be pulled back in a certain, more-or-less unique way. A consequence of its "universality" is that whenever a cohomological identity holds for $f \tau$ it must hold for all $\tau$-maps.

Of course, the cohomological structure of e.g., $X_{0} \tau$ is not clear. The way we can check a cohomological identity for $f \tau$ has two levels: (1) If we know the "structure" of the identity, and only concrete coefficients are missing, then we can restrict it to the zero sections $K_{0}(\eta)$ of the global normal form of $\eta$. Usually the computations can be carried out easily there. (2) If we also need to prove the identity for $f \tau$ then we need a Mayer-Vietoris argument, which is usually easy in the complex case, for more details see $[\mathbf{R}]$.

\section{Morin singularities.}

The prototype of the Morin singularity $A_{i}$ in the case $M^{*} \longrightarrow N^{*+1}$ is the miniversal unfolding of the map $\left(\mathbb{C}^{1}, 0\right) \longrightarrow\left(\mathbb{C}^{2}, 0\right), x \mapsto\left(x^{i+1}, 0\right)$, namely

$$
\begin{aligned}
\left(x, y_{1}, \ldots, y_{i-1}, z_{1}, \ldots, z_{i}\right) & \\
& \mapsto\left(x^{i+1}+\sum_{j=1}^{i-1} y_{j} x^{j}, \sum_{j=1}^{i} z_{j} x^{j}, y_{1}, \ldots, y_{i-1}, z_{1}, \ldots, z_{i}\right) .
\end{aligned}
$$


The maximal compact symmetry group is $U(1) \times U(1)$ with the representation $\lambda_{0}=\rho_{1} \oplus \rho_{V}, \lambda_{1}=\rho_{1}^{i+1} \oplus \rho_{2} \oplus \rho_{V}$, where

$$
\rho_{V}=\bigoplus_{j=1}^{i-1} \rho_{1}^{i+1-j} \oplus \bigoplus_{j=1}^{i}\left(\bar{\rho}_{1} \otimes \rho_{2}\right) .
$$

Here $\rho_{1}, \rho_{2}$ are the standard 1-dimensional representations of the 1st and 2nd factor of $U(1) \times U(1)$, and the powers mean tensor powers.

Now we compute the Chern classes of the map $f \tau$ restricted to $K_{0}\left(A_{i}\right)$ :

$$
\begin{aligned}
\left.c(f \tau)\right|_{K_{0}\left(A_{i}\right)} & =\frac{f^{*} c\left(T\left(B G_{A_{i}}\right) \oplus \xi_{1}\left(A_{i}\right)\right)}{c\left(T\left(B G_{A_{i}}\right) \oplus \xi_{0}\left(A_{i}\right)\right)}=\frac{c\left(f^{*}\left(\xi_{1}\left(A_{i}\right)\right)\right)}{c\left(\xi_{0}\left(A_{i}\right)\right)} \\
& =\frac{(1+(i+1) a)(1+b)}{1+a} \\
& =1+(i a+b)+\left(-i a^{2}+i a b\right)+\left(i a^{3}-i a^{2} b\right)+\cdots .
\end{aligned}
$$

It seems to be a more difficult subject to compute $m_{r}(f \tau)$ (= the Poincarédual of the fundamental homology class carried by the closure of $K_{0}\left(r A_{0}\right)$ ) restricted to $K_{0}\left(A_{i}\right)$. This is called the incidence class $I\left(r A_{0}, A_{i}\right)$ of the singularities $r A_{0}$ and $A_{i}$ in [R]. Of course, this is trivially zero if $r>i+1$, since in this case there are no $r$-tuple points near $A_{i}$. It is interesting, that the analogue of this easy observation was enough when computed Thom polynomials, but it is not enough here.

\section{Lemma 4.1.}

$$
\begin{aligned}
\left.m_{r}\right|_{K_{0}\left(A_{i}\right)} & =\left(\begin{array}{c}
i \\
r-1
\end{array}\right)(b-a)(b-2 a) \cdot \ldots \cdot(b-(r-1) a) \\
\left.f \tau^{*}\left(n_{r}\right)\right|_{K_{0}\left(A_{i}\right)} & =\left(\begin{array}{c}
i+1 \\
r
\end{array}\right) b(b-a)(b-2 a) \cdot \ldots \cdot(b-(r-1) a) .
\end{aligned}
$$

Let us concentrate on the computation of $\left.m_{r}\right|_{K_{0}\left(A_{i}\right)}=I\left(r A_{0}, A_{i}\right)$ first. Since this is the first nontrivial incidence class computation, before giving the complete proof let us discuss easier special cases.

Consider the special case of $i=2$. A prototype of $A_{2}$ is

$$
\left(x, y, z_{1}, z_{2}\right) \mapsto\left(x^{3}+y x, z_{1} x+z_{2} x^{2}, y, z_{1}, z_{2}\right),
$$

with the maximal compact symmetry group $U(1) \times U(1)$ acting as

$$
\kappa: \alpha \oplus \alpha^{2} \oplus \bar{\alpha} \beta \oplus \bar{\alpha}^{2} \beta \quad \text { and } \quad \alpha^{3} \oplus \beta \oplus \alpha^{2} \oplus \bar{\alpha} \beta \oplus \bar{\alpha}^{2} \beta
$$

( $\alpha, \beta$ are the standard representations of the first and second $U(1)$, products are meant to be tensor products). Easy analysis of $\kappa$ shows that the closure of the triple point set (in the source) is $z_{1}=0, z_{2}=0$. Considering this set 
in each fibre of $\xi_{0}\left(A_{2}\right)$ we get a subbundle $\xi$, so

$$
\begin{aligned}
\left.m_{3}\right|_{K_{0}\left(A_{2}\right)} & =\left.\left[\operatorname{cl} K_{0}\left(3 A_{0}\right)\right]\right|_{K_{0}\left(A_{2}\right)}=e\left(\xi_{0}\left(A_{2}\right) / \xi\right) \\
& =\operatorname{top}((1+b-a)(1+b-2 a))=(b-a)(b-2 a) .
\end{aligned}
$$

This easy method works if the (closure of the) $r$-tuple point set of the prototype is smooth at 0 . This is hardly ever the case. E.g., the (closure of the) double point set of the same $\kappa$ has the equation $z_{1}^{2}+y z_{2}^{2}=0$. Although it is not smooth at 0 , its tangent cone $z_{1}^{2}=0$ is a linear space with multiplicity 2 , so the Euler class of the subbundle corresponding to $z_{1}$ in $\xi_{0}\left(A_{2}\right)$ has to be counted twice: $\left.m_{2}\right|_{K_{0}\left(A_{2}\right)}=2(b-a)$.

This last computation relied on our ability to write up the (scheme theoretically) "correct equation system" for the $r$-tuple point set (with the appropriate number of equations). This seems to be impossible in general, already in the $r=3, i=3$ case. However we can easily give a parameterization (a desingularization) of the $r$-tuple point set, which gives us a complete proof.

Proof. Consider the map $g: \mathbb{C}^{2 i} \longrightarrow \mathbb{C}^{2 i+1}$

$$
\begin{aligned}
&\left(x, y_{1}, \ldots, y_{i-1}, z_{1} \ldots, z_{i}\right) \\
& \mapsto\left(x^{i+1}+\sum_{j=1}^{i-1} y_{j} x^{j}, \sum_{j=1}^{i} z_{j} x^{j}, y_{1}, \ldots, y_{i-1}, z_{1}, \ldots, z_{i}\right)
\end{aligned}
$$

which is a (representative of a) prototype of $A_{i}$. Let the first two coordinate functions - as functions of $x$ - be denoted by $e_{1}(x)$ and $f_{1}(x)$ The natural parameterization of the closure of the double point set would come from $\left\{(u, v) \in \mathbb{C}^{2 i} \times \mathbb{C}^{2 i} \mid g(u)=g(v)\right\}$, but of course there is no need to "double" the unfolding parameters $y_{1}, \ldots, y_{i-1}, z_{1}, \ldots, z_{i}$. Also, we have to get rid of the diagonal component, so we consider the set

$$
\begin{aligned}
\left\{\left(x_{1}, x_{2}, y_{1}, \ldots, y_{i-1}, z_{1}, \ldots, z_{i}\right) \mid e_{2}\left(x_{1}\right):=\right. & \frac{e_{1}\left(x_{1}\right)-e_{1}\left(x_{2}\right)}{x_{1}-x_{2}}, \\
& \left.f_{2}\left(x_{1}\right):=\frac{f_{1}\left(x_{1}\right)-f_{1}\left(x_{2}\right)}{x_{1}-x_{2}}\right\} .
\end{aligned}
$$

On one hand this set is smooth - it is a graph of a map $\left(x_{1}, x_{2}, y_{2}, \ldots, y_{i-1}\right.$, $\left.z_{2}, \ldots, z_{i}\right) \mapsto\left(y_{1}, z_{1}\right)$, on the other hand forgetting $x_{2}$ it projects to the closure of the double point set of $g$. So we get the following desingularization of the closure of the double point set:

$$
\left(x_{1}, x_{2}, y_{2}, \ldots, y_{i-1}, z_{2}, \ldots, z_{i}\right) \mapsto\left(x_{1}, Y_{1}, y_{2}, \ldots, y_{i-1}, Z_{1}, z_{2}, \ldots, z_{i}\right),
$$

where the function $Y_{1}$ is from $-x_{2}^{i}+\mathfrak{m}\left(x_{1}, y_{2}, \ldots, y_{i-1}, z_{2}, \ldots, z_{i}\right)$ and the function $Z_{1}$ is from $\mathfrak{m}\left(x_{1}, y_{2}, \ldots, y_{i-1}, z_{2}, \ldots, z_{i}\right)$. Here $\mathfrak{m}$ denoted the maximal ideal in the function algebra in the variables given in brackets. Now 
it can be easily read off that the tangent cone of the closure of the double point set is the subspace spanned by the coordinates

$$
\left(x_{1}, y_{1}, \ldots, y_{i-1}, z_{2}, \ldots, z_{i}\right)
$$

with multiplicity $i$. So we get that $\left.m_{2}\right|_{K_{0}\left(A_{i}\right)}=i \cdot e\left(\right.$ the subbundle of $\xi_{0}\left(A_{i}\right)$ corresponding to $\left.z_{1}\right)=i(b-a)$.

Now we turn to the closure of the triple point set, so we consider

$$
\begin{aligned}
&\left\{\left(x_{1}, x_{2}, x_{3}, y_{1}, \ldots, y_{i-1}, z_{1}, \ldots, z_{i}\right) \mid e_{2}\left(x_{1}\right), f_{2}\left(x_{1}\right)\right. \\
&\left.e_{3}\left(x_{1}\right):=\frac{e_{2}\left(x_{1}\right)-e_{2}\left(x_{3}\right)}{x_{1}-x_{3}}, f_{3}\left(x_{1}\right):=\frac{f_{2}\left(x_{1}\right)-f_{2}\left(x_{3}\right)}{x_{1}-x_{3}}\right\} .
\end{aligned}
$$

This is again smooth - a graph of a map to the coordinates $y_{1}, z_{1}, y_{2}, z_{2}$-, and parametrizes the closure of the triple point set by forgetting $x_{2}$ and $x_{3}$. By calculation we get that the closure of the triple point set is parametrized by

$$
\begin{aligned}
\left(x_{1}, x_{2}, x_{3}, y_{3}, \ldots, y_{i-1}, z_{3}, \ldots, z_{i}\right) & \\
& \mapsto\left(x_{1}, Y_{1}, Y_{2}, y_{3}, \ldots, y_{i-1}, Z_{1}, Z_{2}, z_{3}, \ldots, z_{i}\right),
\end{aligned}
$$

where the functions $Z_{1}, Z_{2}$ are zero - modulo $\mathfrak{m}\left(x_{1}, y_{3}, \ldots, y_{i-1}, z_{3}, \ldots, z_{i}\right)$ and the functions $Y_{1}, Y_{2}$ are homogeneous degree $i$ and $i-1$ polynomials in $x_{2}, x_{3}$ - modulo the mentioned maximal ideal. Again, we can read off that the tangent cone of the closure of the triple point set is the subspace spanned by the coordinates

$$
\left(x_{1}, y_{1}, \ldots, y_{i-1}, z_{3}, \ldots, z_{i}\right)
$$

with multiplicity $i(i-1)$. So we get that

$$
\begin{aligned}
\left.m_{3}\right|_{K_{0}\left(A_{i}\right)} & =\frac{i(i-1)}{2} \cdot e\left(\text { the subbundle of } \xi_{0}\left(A_{i}\right) \text { corresponding to } z_{1}, z_{2}\right) \\
& =\left(\begin{array}{c}
i \\
2
\end{array}\right)(b-a)(b-2 a) .
\end{aligned}
$$

Going on like this we obtain a (degree $(r-1)$ !) parameterization of the closure of the $r$-tuple set as

$$
\begin{aligned}
\left(x_{1}, \ldots, x_{r}, y_{r}, \ldots, y_{i-1}, z_{r}, \ldots, z_{i}\right) & \\
& \mapsto\left(x_{1}, Y_{1}, \ldots, Y_{r-1}, y_{r}, \ldots, y_{i-1}, Z_{1}, \ldots, Z_{r-1}, z_{r}, \ldots, z_{i}\right),
\end{aligned}
$$

where the functions $Z_{1}, \ldots, Z_{r-1}$ are in the maximal ideal generated by the coordinates $x_{1}, y_{r}, \ldots, y_{i-1}, z_{r}, \ldots, z_{i}$, while $Y_{1}, \ldots, Y_{r-1}$ are homogeneous 
degree $i, i-1, \ldots, i-r+2$ polynomials in $x_{2}, x_{3}, \ldots, x_{r}$. So the sought incidence class $\left.m_{r}\right|_{K_{0}\left(A_{i}\right)}$ is

$$
\begin{aligned}
& \frac{i(i-1) \ldots(i-r+2)}{(r-1) !} \cdot e\left(\text { the subbundle of } \xi_{0}\left(A_{i}\right)\right. \text { corresponding } \\
& \left.\quad \text { to } z_{1}, \ldots, z_{r-1}\right)=\left(\begin{array}{c}
i \\
r-1
\end{array}\right)(b-a)(b-2 a) \ldots(b-(r-1) a),
\end{aligned}
$$

which was to be proved.

To prove the second equality we use the Gysin push-forward $f_{*}$ in cohomology, and its well-known property $f_{*} f^{*} v=v \cup f_{*} 1$ for all $v$. Let us apply this for $f=f\left(A_{i}\right)$, and pull it back by $f$ :

$$
f^{*} f_{*}\left(f^{*} v\right)=\left(f^{*} v\right) \cup f^{*}\left(n_{1}\right) .
$$

Since $f^{*}$ is isomorphism, we can put any cohomology class for $f^{*} v$, e.g., $m_{r}$ :

$$
f^{*} f_{*} m_{r}=m_{r} \cup f^{*} n_{1} .
$$

The left hand side is clearly $r \cdot f^{*} n_{r}$ and $f^{*}\left(n_{1}\right)=(i+1) b$ (with the same method - it is given by parameterization). This implies the second equality.

As an example let us show how to compute $p_{1}$. This must be weighted homogeneous of degree 2 , so it is a constant $A$ times $c_{1}$. Consider the first multiple-point formula $m_{2}=f^{*} n_{1}-A c_{1} m_{1}$ for $f=f \tau$ (with $\tau$ large enough to consist $\left.A_{0}\right)$ and restrict it to $K_{0}\left(A_{0}\right)$. According to Lemma 4.1 and the Chern-class computation above it, the restrictions of $m_{2}, f^{*} n_{1}$ and $c_{1}(f \tau)$ to $K_{0}\left(A_{1}\right)$ are $\left(\begin{array}{l}0 \\ 1\end{array}\right)(b-a),\left(\begin{array}{l}1 \\ 1\end{array}\right) b, b$ respectively. So our formula reduces to $0=b-A \cdot b \in H^{1}\left(B G_{A_{0}}\right)=\mathbb{Z}[a, b]$ (the equality is meant modulo torsion, but there is no torsion at all here). So $A=1$, i.e., $p_{1}=c_{1}$.

To compute $p_{2}=A c_{1}^{2}+B c_{2}$ we proceed similarly. Knowing the result above, restrict the triple point formula $m_{3}=f^{*} n_{2}-c_{1} m_{2}+\left(A c_{1}^{2}+\right.$ $\left.B c_{2}\right) m_{1}$ (applied to $f \tau$, where $\tau$ big enough to consist $A_{1}$ ) to $K_{0}\left(A_{1}\right)$. Using Lemma 4.1 and the Chern-class computation above it again, we get

$$
\begin{aligned}
\left(\begin{array}{l}
1 \\
2
\end{array}\right)(b-a)(b-2 a)= & \left(\begin{array}{l}
2 \\
2
\end{array}\right) b(b-a)-(a+b)\left(\begin{array}{l}
1 \\
1
\end{array}\right)(b-a) \\
& +\left(A(a+b)^{2}+B\left(-a^{2}+a b\right)\right) \in \mathbb{Z}[a, b],
\end{aligned}
$$

which gives an (overdetermined) system of linear equations on $A, B$, with the unique solution $A=0, B=1$. Therefore $p_{2}=c_{2}$.

To obtain the next polynomial $p_{3}=A c_{1}^{3}+B c_{1} c_{2}+C c_{3}$ we use the values of $p_{1}, p_{2}$ just computed and restrict formula $\left(1_{4}\right)$ (applied to an appropriate 
$f \tau)$ to $K_{0}\left(A_{2}\right)$. We get the equation

$$
\begin{aligned}
0= & b(b-a)(b-2 a)-(3 a+b)(b-a)(b-2 a)+\left(-3 a^{2}+3 a b\right) 2(b-a) \\
& -\left(A(3 a+b)^{3}+B(3 a+b)\left(-3 a^{2}+3 a b\right)+C\left(3 a^{3}-3 a^{2} b\right)\right)
\end{aligned}
$$

in $\mathbb{Z}[a, b]$ which leads to an (overdetermined) system of linear equations with the unique solution $A=0, B=1, C=3$, so $p_{3}=c_{1} c_{2}+3 c_{3}$.

We can go on like that: When trying to compute $p_{i}$ we use the already computed $p_{j}$ 's $(j<i)$ and restrict formula $\left(1_{i+1}\right)$ (when applied to an appropriate $f \tau$ ) to $K_{0}\left(A_{i-1}\right)$. We obtain a system of linear equations on the coefficients of $p_{i}$. From $i=6$ the solution is not unique, contains a certain number of parameters - at which we are not surprised, see Remark 2.2.

Remark 4.2. By now it is clear how to compute the polynomials $p_{i}$, once we know that formula $\left(1_{r}\right)$ holds (for corank 1 maps) with some polynomials. Of course, our method is also capable to prove that the formulas, with the computed polynomials really hold for corank 1 maps. For this we need to check three things.

First that they hold if restricted to $K_{0}\left(A_{i}\right)$ for any $i$, not just the small ones we used when determined the coefficients. This can be done individually (e.g., for $\left(1_{2}\right)$ we need to check that $i(b-a)=(i+1) b-(i a+b)$ holds for any $i$ ), or using a general argument, based on the fact that if a polynomial (in $i$ ) vanishes in $i=0,1, \ldots, \mathrm{deg}$, then it vanishes for greater $i$ 's, too, since it must be the 0 polynomial.

Secondly we need to check that the formulas hold restricted to $K_{0}(\eta)$ 's, where $\eta$ is a linear combination of $A_{i}$ 's. The method we used in $[\mathbf{R}]$ (when dealing with immersion formulas) shows that modulo torsion this task can be reduced to verifying the formulas restricted to $K_{0}(\zeta)$ 's, where the $\zeta$ 's are the mono-singularities occurring in $\eta$.

Thirdly we need to use a Mayer-Vietoris argument to deduce the following: If e.g., $\left(1_{r}\right)$ hold restricted to all blocks in $X_{0} \tau$ then it holds in the cohomology of $X_{0} \tau$, too. In the complex case these arguments are easy, see $[\mathbf{R}]$.

\section{Singularities of higher corank.}

Now consider the simplest singularity - denoted as $\mathrm{III}_{2,2}$ by Mather — of Thom-Boardman class $\Sigma^{2,0}$, i.e., the one with local algebra $\mathbb{C}[[x, y]] /\left(x^{2}, y^{2}\right.$, $x y)$. Its prototype is the miniversal unfolding of $(x, y) \mapsto\left(x^{2}, y^{2}, x y\right)$. From this we can see that the maximal compact symmetry group $G_{\mathrm{III}_{2,2}}$ is $U(2)$. So, according to our procedures we should now look for a miniversal unfolding which admits $U(2)$ as a (right-left linear) symmetry group. Of course, such a prototype could be given, but the calculation is much simpler if we 
choose

$$
\kappa:\left(x, y, u_{1}, u_{2}, v_{1}, v_{2}\right) \mapsto\left(x^{2}+u_{1} x+u_{2} y, y^{2}+v_{1} x+v_{2} y, x y, u_{1}, u_{2}, v_{1}, v_{2}\right)
$$

as our prototype. On this germ only $U(1) \times U(1)$ (the maximal torus of $U(2))$ acts linearly, with the representations

$$
\lambda_{0}\left(\mathrm{III}_{2,2}\right)=\alpha \oplus \beta \oplus \mu_{V} \quad \lambda_{1}\left(\mathrm{III}_{2,2}\right)=\alpha^{2} \oplus \beta^{2} \oplus \alpha \beta \oplus \mu_{V}
$$

where $\mu_{V}=\alpha \oplus \alpha^{2} \bar{\beta} \oplus \bar{\alpha} \beta^{2} \oplus \beta$ ( $\alpha, \beta$ are the standard 1-dimensional representations of the first and the second $U(1))$. The reduction of the maximal symmetry group to a smaller one usually causes loss of information, but not now, as we will see below.

To obtain the equation of the (closure of the) double point set

$$
\begin{aligned}
& \left\{\left(x, y, u_{1}, u_{2}, v_{1}, v_{2}\right) \mid \exists\left(x_{1}, y_{1}\right) \neq(x, y)\right. \text { s.t. } \\
& \left.\kappa\left(x, y, u_{1}, u_{2}, v_{1}, v_{2}\right)=\kappa\left(x_{1}, y_{1}, u_{1}, u_{2}, v_{1}, v_{2}\right)\right\}
\end{aligned}
$$

in the source of $\kappa$ let $\lambda$ be $x_{1} / x=y / y_{1}$, i.e., let $x_{1}=\lambda x, y_{1}=\frac{1}{\lambda} y$. Then the equation of the double point set is the resultant of the two polynomials

$$
\frac{\kappa_{1}(x, y)-\kappa_{1}\left(\lambda x, \frac{1}{\lambda} y\right)}{(\lambda-1)}, \quad \frac{\kappa_{2}(x, y)-\kappa_{2}\left(\lambda x, \frac{1}{\lambda} y\right)}{(\lambda-1)} .
$$

These two polynomials are (by calculation)

(*) $\lambda^{2}\left(-x^{2}\right)+\lambda\left(-u_{1} x-x^{2}\right)+\left(u_{2} y\right), \quad \lambda^{2}\left(-v_{1} x\right)+\lambda\left(v_{2} y+y^{2}\right)+\left(y^{2}\right)$.

Their resultant (after getting rid of the "false" factor $x^{2} y^{2}$ ) is

$$
\begin{aligned}
& -\left(v_{1} x^{3}+u_{2} y^{3}\right)-\left(v_{2} x^{2} y+u_{1} x y^{2}\right)-\left(2 u_{1} v_{1} x^{2}+2 u_{2} v_{2} y^{2}\right) \\
& -\left(u_{1} v_{2} x y\right)-\left(3 v_{1} u_{2} x y\right)-\left(u_{1}^{2} v_{1} x+u_{2} v_{2}^{2} y\right) \\
& -\left(u_{2} v_{1} v_{2} x+u_{1} u_{2} v_{1} y\right)+\left(u_{2}^{2} v_{1}^{2}\right)-\left(u_{1} u_{2} v_{1} v_{2}\right) .
\end{aligned}
$$

This set coincides with its own tangent cone, which is now not a linear space (with multiplicity), so we cannot use the methods above to compute the cohomology class $m_{2}$ represented by the double points of the map $f_{\kappa}$ : $\xi_{0} \longrightarrow \xi_{1}$ (recall that $\xi_{0}, \xi_{1}$ are vector bundles over $U(1) \times U(1)$ associated with the universal principal $U(1) \times U(1)$-bundle using the representations $\lambda_{1}, \lambda_{2}$, and the map is fibrewise equivalent to $\left.\kappa\right)$. Our method now is the following. The class sought is from $H^{2}(B U(1) \times B U(1))=\mathbb{Z} \oplus \mathbb{Z}$, so it is $A a+B b$, where $a$ and $b$ correspond to the two $U(1)$ 's. Let us consider a 2 -cycle in $B U(1) \times B U(1)$, on which $a$ takes the value 1 , by abuse of language let us call this 2-cycle also $a$. If we calculate the value of $m_{2}$ on this 2-cycle, we get the coefficient $A$. Let us take a general perturbation of $a$ in $\xi_{0}$. Then the coefficient $A$ above will be the intersection number of this perturbation and the (closure of the) double-point locus of $f_{\kappa}$. However we can make this perturbation in a special way: We can lift $a$ in a universal direction (not contained in the double-point locus) everywhere but in one 
point. The lifting will have a boundary over this point - the image of $S^{1} \subset \mathbb{C}$ under the map: $z \mapsto\left(z, 1, z, z^{2}, \bar{z}, 1\right)$ - see the representation $\lambda_{0}\left(\mathrm{III}_{2,2}\right)$. To obtain a complete lifting (perturbation) of $a$, we have to extend this map from $S^{1}$ to the disc. This way we achieved that the intersection number of the double point locus and (a perturbation of) $a$ is to be counted in one fibre. Indeed we only have to calculate the intersection number of the curve $D^{2} \longrightarrow \mathbb{C}^{6}, z \mapsto\left(z, 1, z, z^{2}, \bar{z}, 1\right)$ and the double point locus, which is given by an equation above. To get this we substitute $x=z, y=1, u_{1}=$ $z, u_{2}=z^{2}, v_{1}=\bar{z}, v_{2}=1$ in the equation. We get $z^{2}\left(|z|^{4}-10|z|^{2}-7\right)=0$. Since we can clearly get rid of the second factor by a small perturbation, we obtain that the intersection number is (so $A$ equals to) 2. Very similarly, we substitute $x=1, y=z, u_{1}=1, u_{2}=\bar{z}, v_{1}=z^{2}, v_{2}=z$ into the same equation and we get that $B=2$. So the double point cohomology class represented by the double point locus of $f_{\kappa}$ is $2 a+2 b$.

Remark 5.1. Technically what happened is that we calculated the equation of the double point locus in one fibre and — in some sense - we "substituted the representation" $\lambda_{0}$ for the variables (the value of $A$ and $B$ could have been obtained this way together). Of course, this method works for the singularities $A_{i}$ above, too.

Now let us deal with the (closure of the) triple point set. This is given by the condition that the two equations in $(*)$ have two common roots. This is equivalent to saying that the matrix

$$
\left(\begin{array}{ccc}
-x^{2} & -u_{1} x-x^{2} & u_{2} y \\
-v_{1} x & y^{2}+v_{2} y & y^{2}
\end{array}\right)
$$

has rank $\leq 1$. If this variety (in fact its ideal) were given by 2 equations then we could follow the same procedure as above. This is not the case, but there are ways to work with these kind of determinantal varieties. An easy way which works here is that we can write it as a difference of two varieties both given by two equations.

$\left\{\operatorname{det}\left(\begin{array}{cc}-x^{2} & -u_{1} x-x^{2} \\ -v_{1} x & y^{2}+v_{2} y\end{array}\right), \operatorname{det}\left(\begin{array}{cc}-u_{1} x-x^{2} & u_{2} y \\ y^{2}+v_{2} y & y^{2}\end{array}\right)\right\} \backslash\left\{-u_{1}-x, y+v_{2}\right\}$.

Now we can "substitute the representation $\lambda_{0}\left(\mathrm{III}_{2,2}\right)$ " into these equations and obtain that the cohomology class represented by the (closure of the) triple point locus of $f_{\kappa}$ is $(2 b)(2 a)-a b=3 a b$.

Since there are no quadruple points near the singularity $\mathrm{III}_{2,2}$, the cohomology classes corresponding to multiple points of $f_{\kappa}$ are as follows

$$
m_{1}=1, \quad m_{2}=2 a+2 b, \quad m_{3}=3 a b, \quad m_{\geq 4}=0 .
$$

Again, we can use the Gysin homomorphism to obtain from this classes the cohomology classes corresponding to multiple points in the target (pulled 
back), just as we did in case of $A_{i}$ singularities. We get that $f_{\kappa}^{*}\left(n_{r}\right)=$ $1 / r \cdot 4(a+b) m_{r}$, that is

$$
\begin{gathered}
f_{\kappa}^{*}\left(n_{1}\right)=4(a+b), \quad f_{\kappa}^{*}\left(n_{2}\right)=4 a^{2}+8 a b+4 b^{2}, \\
f_{\kappa}^{*}\left(n_{3}\right)=4 a^{2} b+4 a b^{2}, \quad f_{\kappa}^{*}\left(n_{\geq 4}\right)=0 .
\end{gathered}
$$

We also need the total Chern class of the map $f_{\kappa}$, which can be read from the representations $\lambda_{0}\left(\mathrm{III}_{2,2}\right), \lambda_{1}\left(\mathrm{III}_{2,2}\right)$ as follows

$$
\begin{aligned}
& \frac{(1+2 a)(1+2 b)(1+a+b)}{(1+a)(1+b)} \\
& =1+(2 a+2 b)+(3 a b)+\left(-a^{2} b-a b^{2}\right)+\left(a^{3} b-a^{2} b^{2}+a b^{3}\right) \\
& \quad+\left(-a^{4} b+a^{3} b^{2}+a^{2} b^{3}-a b^{4}\right)+\cdots .
\end{aligned}
$$

Now we have everything to substitute into the formulas $\left(1_{r}\right)$. Concrete calculation shows that $\left(1_{r}\right)$ holds for $f=f_{\kappa}$ if $r=1,2,3,4,5 ;\left(1_{6}\right)$ holds if and only if $t=43$ and $\left(1_{7}\right)$ holds if and only if in addition $u_{2}=2 u_{1}-284$.

Recall that the whole maximal symmetry group of a prototype of $\mathrm{III}_{2,2}$ is $U(2)$ and we worked only with its maximal torus $U(1) \times U(1)$, and found that some formulas hold in $H^{*}(B U(1) \times B U(1))$. However these formulas are the natural images of the analogous formulas for in $H^{*}(B U(2))$, and the map $H^{*}(B U(2)) \longrightarrow H^{*}(B U(1) \times B U(1))$ induced by the inclusion $U(1) \times U(1) \subset U(2)$ is injective. So we can also conclude that formulas $\left(1_{r}\right)$ $r=1,2, \ldots, 7$ hold with $t=43, u_{2}=2 u_{1}-284$ in the cohomology of the base space of the global normal form of $\mathrm{III}_{2,2}$.

This means that if a formula like $\left(1_{r}\right)$ holds for maps with $\mathrm{III}_{2,2}$ singularity then they must have the mentioned coefficients. To argue that they really hold for every map not having worse singularity than $\mathrm{III}_{2,2}$ we would need that these formulas hold restricted to the base spaces of the global normal forms of multi-singularities, where the multi-singularity is put together from mono-singularities not worse than $\mathrm{III}_{2,2}$. Easy cohomological analysis shows that if a formula holds in the base spaces of the global normal form of some mono-singularities, then the analogous formula holds (at least modulo torsion) in the base space of the global normal form of a multi-singularity put together from these mono-singularities.

According to the universal property of the gPTc this means that formulas $\left(1_{r}\right)$ for $r=2,3,4,5,6(t=42), 7\left(u_{2}=2 u_{1}-284\right)$ hold for any map not having more complicated singularity than $\mathrm{III}_{2,2}$.

Now, if we want to set the value of the parameter $u_{1}$ (and so $u_{2}$ ) we need to go through the analogous procedure with the stable singularities of codimension 7 . In fact there is only one such, the one denoted by Mather as $\mathrm{I}_{2,2}$ 
(it is defined by its local algebra $\mathbb{C}[[x, y]] /\left(x y, x^{2}+y^{2}\right)$ ). Recall that the procedure involves the following: Determination of the maximal compact symmetry group (or in some cases at least its maximal torus), its representations on the source and target spaces - then we have the global normal form. From this we can compute the total Chern class (basically $c=c\left(\lambda_{1}-\lambda_{0}\right)$ ). More difficult is the determination of the cohomology classes represented by the multiple points - i.e., the incidence classes $I\left(r A_{0}, \mathrm{I}_{2,2}\right)$. However, the methods we used in the case of $A_{i}, \mathrm{III}_{2,2}$ are sufficient to go through the calculation with $\mathrm{I}_{2,2}$ (which we omit here), and get that

$$
\begin{gathered}
\text { the total Chern class }=\frac{(1+2 a)(1+2 b)(1+c)}{(1+a)(1+b)} \quad \in \mathbb{Z}[a, b, c] \text { and } \\
\qquad m_{1}=1, \quad m_{2}=3 c-a-b, \\
m_{3}=3 c^{2}-3(a+b) c+3 a b, \quad m_{4}=(c-a)(c-b)(c-a-b) .
\end{gathered}
$$

As above we can substitute these values, and prove Theorem 2.3.

\section{Remarks.}

Let us make some remarks. First it would be nice to prove the so called refined formulas of Kleiman [K4] with our method, i.e., proving the multiplepoint formulas without the comment "modulo torsion". Although this does not seem to be impossible, we should make a much finer cohomologial analysis, already in the case of immersion formulas - so cohomologies of the symmetric groups will get in the picture.

Another interesting question is the existence of analogous multiple-point formulas over the reals. The prototype of these formulas has been found by Szücs in $[\mathbf{S z 2}]$ and $[\mathbf{S z 3}]$. The fact is that our formulas can be translated blindly to the real world $\left(c_{i} \mapsto w_{i}\right)$ with the only difference that $f^{*}\left(n_{r}\right)$, which does not make sense there should be replaced by suitable "linking cohomology class", more details in a subsequent paper.

In $[\mathbf{R}]$ we computed Thom polynomials of mono-singularities no matter how complicated they are. Here we computed "Thom polynomials" of multisingularities, whose mono-terms are all the simplest ones: $A_{0}$. So there is gap in between. One should understand and compute the Thom polynomials of multi-singularities of type, e.g., $A_{1}+A_{1}, 2 A_{0}+3 A_{1}+\mathrm{III}_{2,2}$, etc. The author believes that these problems also can be attacked by the method presented in this paper.

\section{References}

[AVGL] V. Arnold, V. Vasil'ev, V. Goryunov and O. Lyashko, Singularities. Local and Global Theory, Enc. Math. Sci., Vol. 6. (Dynamical Systems VI), Springer-Verlag, 1993, MR 91h:58010b, Zbl 0787.58001. 
[C1] S.J. Colley, Enumerating stationary multiple-point, Adv. in Math., 66(2) (1987), 149-170, MR 89g:14042, Zbl 0652.14023.

[C2] Coincidence formulas for line complexes, Comm. in Algebra, 16(11) (1988), 2363-2385, MR 90e:14058, Zbl 0661.14039.

[H] R.J. Herbert, Multiple points of immersed manifolds, Mem. Amer. Math. Soc., 34(250) (1981), MR 84c:57022, Zbl 0493.57012.

[J] T. Johnsen, Eight-secant conics for space curves, Math. Z., 211 (1992), 609-626, MR 93k:14075, Zbl 0759.14042.

[Ka1] S. Katz, Lines on complete intersection threefolds, Comp. Math., 60 (1986), 151162, MR 87c:14045, Zbl 0563.14020.

[Ka2] Iteration of multiple point formulas and applications to conics, in "Algebraic Geometry — Sundance, 1986", A. Holme, R. Speiser, eds., SLNM, 1311 (1988), 147-155, MR 89j:14032, Zbl 0657.14035.

[K1] S.L. Kleiman, The enumerative theory of singularities, in "Real and Complex Singularities" (Proc. Conf., Oslo 1976), P. Holm, ed., Sijthoff and Noordhoff, (1977), 297-396, MR 58 \#27960, Zbl 0385.14018.

[K2] _ Multiple point formulas for maps, in "Enumerative Geometry and Classical Algebraic Geometry" (P. Barz, Y. Hervier eds.), Birkhauser, Prog. in Math., 24 (1982), 237-252, MR 84j:14059, Zbl 0503.14023.

[K3] - Multiple-point formulas I: Iteration, Acta Math., 147 (1981), 13-49, MR 83j:14006, Zbl 0479.14004.

[K4] ( Multiple-point formulas II: The Hilbert scheme, in "Enumerative Geometry", Proceedings, Sitges 1987, SLNM, 1436, 101-138, MR 92a:14062, Zbl 0714.14036.

[MM] W.L. Marar and D. Mond, Multiple point schemes for corank 1 maps, J. London Math. Soc., 39(2) (1989), 553-567, MR 91c:58010, Zbl 0691.58015.

[Ra] Z. Ran, Curvilinear enumerative geometry, Acta Math., 155(1-2) (1985), 81-101, MR 86m:14040, Zbl 0578.14046.

[R] R. Rimányi, Thom polynomials, symmetries and incidences of singularities, Invent. Math., 143(3) (2001), 499-521, MR 2001k:58082.

[RSz] R. Rimányi and A. Szücs, Generalized Pontrjagin-Thom construction for maps with singularities, Topology, 37(6) (1998), 1177-1191, MR 99g:57031, Zbl 0924.57035.

[Ro] F. Ronga, On multiple points of smooth immersions, Comment. Math. Helv., 55 (1980), 521-527, MR 82d:57018, Zbl 0457.57013.

[Sz1] A. Szücs, Cobordism group of l-immersions I, II (in Russian), Acta Math. Acad. Sci. Hungar., 27 (1976) 343-358; 28 (1976) 93-102, MR 55 \#13431, MR 55 \#13432.

[Sz2] _ Multiple points of singular maps, Math. Proc. Camb. Phyl. Soc., 100 (1986) 331-346, MR 88a:57062, Zbl 0625.57017. 
[Sz3] _ The linking number of singular maps, Comment. Math. Helv., 61 (1986) 360-369, MR 88a:57061, Zbl 0602.57021.

Received February 15, 2000. This research was partially supported by OTKA T029759, FKFP 0226/99.

Department of Analysis

EOTVOS UNIVERSITY

Budapest RÁKóCZi út 5

HUNGARY

E-mail address: rimanyi@cs.elte.hu 